\title{
Dynamic channel properties based on diffuse scattering from vehicles for high frequency bands in NLOS urban environments
}

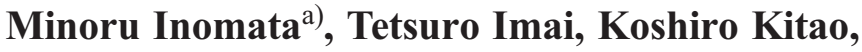 \\ and Yukihiko Okumura \\ NTT DOCOMO, INC., \\ 3-6 Hikarino-oka, Yokosuka-shi, Kanagawa 239-8536, Japan \\ a)minoru.inomata.gn@nttdocomo.com
}

\begin{abstract}
We describe dynamic channel properties based on diffuse scattering from vehicles for high frequency bands in a non-line-of-sight (NLOS) urban environment. We investigate the dominant paths based on the measured power delay angular profile in experiments using a $20-\mathrm{GHz}$ band channel sounder. Based on the results, we clarify that arrival waves from buildings and diffuse scattering from vehicles represent dominant paths in NLOS urban environments. Also, we find that the fading of scattering paths from vehicles is approximately $26 \mathrm{~dB}$ in those paths between birth and death.
\end{abstract}

Keywords: $5 \mathrm{G}$ mobile communication systems, high frequency bands, NLOS urban environments, dynamic channel property

Classification: Antennas and Propagation

\section{References}

[1] NTT DOCOMO, INC., "DOCOMO 5G white paper, 5G radio access: Requirements, concept and technologies," July 2014.

[2] M. Inomata, T. Imai, K. Kitao, Y. Okumura, M. Sasaki, and Y. Takatori, "Radio propagation prediction for high frequency bands using hybrid method of raytracing and ER model with point cloud of urban environments," 12th European Conference on Antennas and Propagation (EuCAP), London, UK, April 2018. DOI:10.1587/transcom.2017EBP3436

[3] 3GPP, TR 38.901 V14.0.0, "Study on channel model for frequencies from 0.5 to $100 \mathrm{GHz}$ (Release 14)," March 2017.

[4] White paper on "5G channel model for bands up to $100 \mathrm{GHz}$," Global Communications Conference, 3rd Workshop on Mobile Communications in Higher Frequency Bands, Washington DC, U.S.A., May 2016 (http://www. 5gworkshops.com/5GCM.html).

[5] ITU-R M.2412-0, "Guidelines for evaluation of radio interface technologies for IMT-2020,” Oct. 2017. 


\section{Introduction}

The amount of traffic in wireless communication systems has been rapidly increasing in recent years [1]. One avenue of research being pursued to address this problem is applying frequency bands above $6 \mathrm{GHz}$ to the next generation mobile communications systems (5G) [1]. These frequency bands include the millimeter wave bands, which use a wider frequency bandwidth and provide attractive higher data rates. Presently, it is assumed that the main service areas for $5 \mathrm{G}$ will be in line-of-sight (LOS) urban environments. However, in terms of deployment cost, it is also desirable to be able to cover non-line-of-sight (NLOS) environments. In addition, a massive multiple-input multiple-output (MIMO) technique using a large number of antenna elements is being investigated to compensate for the large propagation loss in high frequency bands, and the application of beamforming is being considered to obtain a higher antenna gain. Therefore, in order to evaluate these techniques, it is necessary to clarify dynamic channel properties based on the effect of moving objects around the mobile station in a NLOS urban environment.

Since the wavelength is shorter in high frequency bands, diffuse scattering due to various scattering objects such as buildings, roadside trees, road signs, and vehicles must be considered. There have been several studies on diffuse scattering $[2,3,4,5]$. Reference [2] described the effect of diffuse scattering on channel properties in a LOS urban environment. Also, the shielding effect of vehicles and humans on channel properties is described in $[3,4,5]$, and these studies report on a prediction method based on those effects. However, it is not clear how diffuse scattering from moving objects affects the dynamic channel properties in NLOS urban environments. Therefore, we investigate the dynamic channel properties based on diffuse scattering from moving objects in high frequency bands in a NLOS urban environment and clarify the dominant paths based on the measured power delay angular profile using a $20-\mathrm{GHz}$ band channel sounder.

\section{Measurement campaign}

Fig. 1(a) is a photograph of the measurement area in Japan, around Tokyo station. Fig. 1(b) shows the measurement point. To analyze diffuse scattering in high frequency bands, we set the measurement frequencies to the $19.85 \mathrm{GHz}$ band. The transmitter (Tx) antenna is an omni-directional antenna and the receiver (Rx) antenna is a planar array antenna that has 256 elements. An OFDM signal is transmitted from the Tx antenna, which is established on the roof of a vehicle positioned along the roadside. The Rx antenna is affixed to a pole mounted on the car. The Tx antenna height is $2.5 \mathrm{~m}$ and the $\mathrm{Rx}$ antenna height is $5 \mathrm{~m}$. The Tx antenna and the $\mathrm{Rx}$ antenna is placed as shown in Fig. 1(b). The Tx signal power is $30 \mathrm{dBm}$ and the bandwidth is $50 \mathrm{MHz}$. For the measurements, in order to obtain data from 360 degrees in the horizontal plane, the planar array antenna records measurements in 3 directions: 0, 90 and -90 degrees. For data processing, the angular delay profile is obtained using the IFFT and beamforming processing in three different $\mathrm{Rx}$ directions. The three angular delay profiles are concatenated based on the azimuth angle. The profile of the maximum power value of the 180- 
degree elevation angle for each propagation delay is extracted. Then, the power delay profile is obtained by extracting the maximum power value of the azimuth angle for each propagation delay. The angular profile is obtained by extracting the maximum power value of the propagation delay for each azimuth angle. In order to obtain the angular profile, we use beamforming processing on the received signal. The recording duration per direction is $30 \mathrm{~s}$ and 15 snapshots of the power angular delay profiles are obtained.

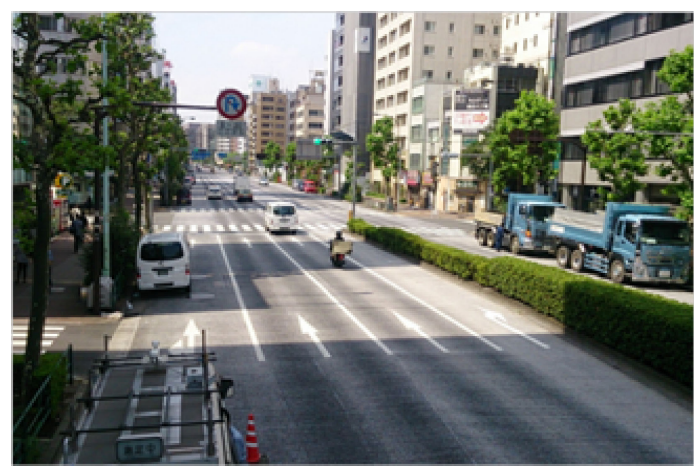

(a) Photo from Rx antenna

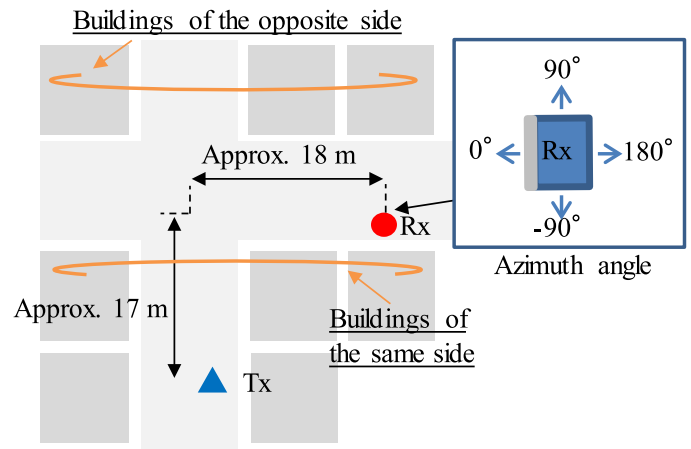

(b) Measurement point

Fig. 1. Measurement environment.

\section{Investigation of dynamic channel properties}

In order to evaluate the dynamic channel properties based on diffuse scattering from the moving objects, we compared the 15 snapshots of the power angular delay profiles and analyzed the time fading characteristics. Fig. 2(a) shows the measurement power delay profile. In the figure, all 15 snapshots of the power delay profiles are represented. From Fig. 2(a), we confirm (1) 1-bounce reflection and then the diffraction at the building in the same lane as the Rx antenna, (2) the diffraction and then 1-bounce reflection at the building in the opposite lane, and (3) the 2-bounce reflections. The 2-bounce reflections represent a propagation path that is first reflected from the building opposite the Rx antenna, reflected from the building on the same side as the Rx antenna, and then arrives at the Rx antenna. We find that the time fading of paths (1), (2), and (3) is relatively stable; however, the fading of the paths at the propagation distance of $47 \mathrm{~m}$ is relatively greater. The fading of 
paths (1), (2) and (3) is approximately $3 \mathrm{~dB}$. On the other hand, the fading of the scattering paths between (1) and (2) is approximately $7 \mathrm{~dB}$. Since these scattering paths are between the path (1) and (2), and the scattering paths fade in accordance with the time variation, we assume that these scattering paths occur from vehicles on the road. In order to analyze the arrival angle of the scattering paths from vehicles, we analyze the angular profiles. Fig. 2(b) shows the angular profiles we obtained. The angular profiles at the propagation distance of $47 \mathrm{~m}$ are extracted and 15 snapshots of the angular profiles are shown. From Fig. 2(b), we confirm that the scattering paths from vehicles arrive from the direction of -50 degrees. This indicates that based on the propagation distance of the scattering paths from the vehicles and the arrival angle, the scattering paths from the vehicles are first reflected from the buildings around the Tx antenna, scattered from the vehicle, reflected from the building on the same side as the $\mathrm{Rx}$ antenna, and arrive at the $\mathrm{Rx}$ antenna. In order to obtain the time fading characteristics, Figs. 2(c) and 2(d) show the power angular delay profiles at 2 different snapshots, 3 and 4, that we obtained over a $2 \mathrm{~s}$ measurement duration, respectively. At the propagation distance of $47 \mathrm{~m}$ and the arrival angle of -50 degrees, we find that the scattering paths from the vehicles are born and die between each snapshot. Fig. 2(e) shows the time fading of the received power at the propagation distance of $47 \mathrm{~m}$ and the arrival angle of -50 degrees. As a result, we find that the fading of the received power is approximately $26 \mathrm{~dB}$ when those paths are born and die. Fig. 3 shows the dominant paths in a NLOS urban environment. Based on these results, we confirm that (1) 1-bounce reflection and then the diffraction at the building in the same lane as the Rx antenna, (2) the diffraction and then 1-bounce reflection at the building in the opposite lane, (3) the 2-bounce reflections, and the scattering path from the vehicles on the road are dominant for high frequency bands in a NLOS urban environment.

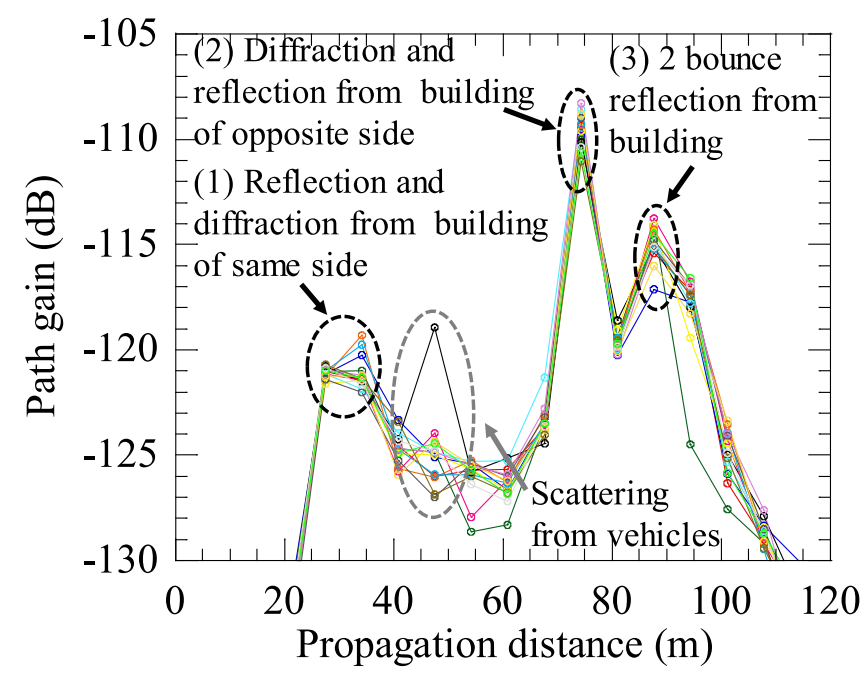

(a) Power delay profiles 


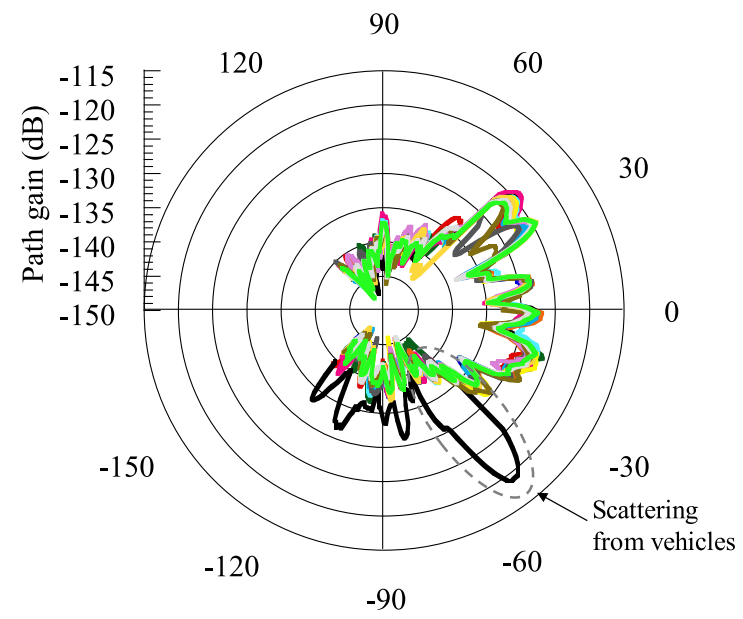

(b) Angular profiles at propagation distance of $47 \mathrm{~m}$

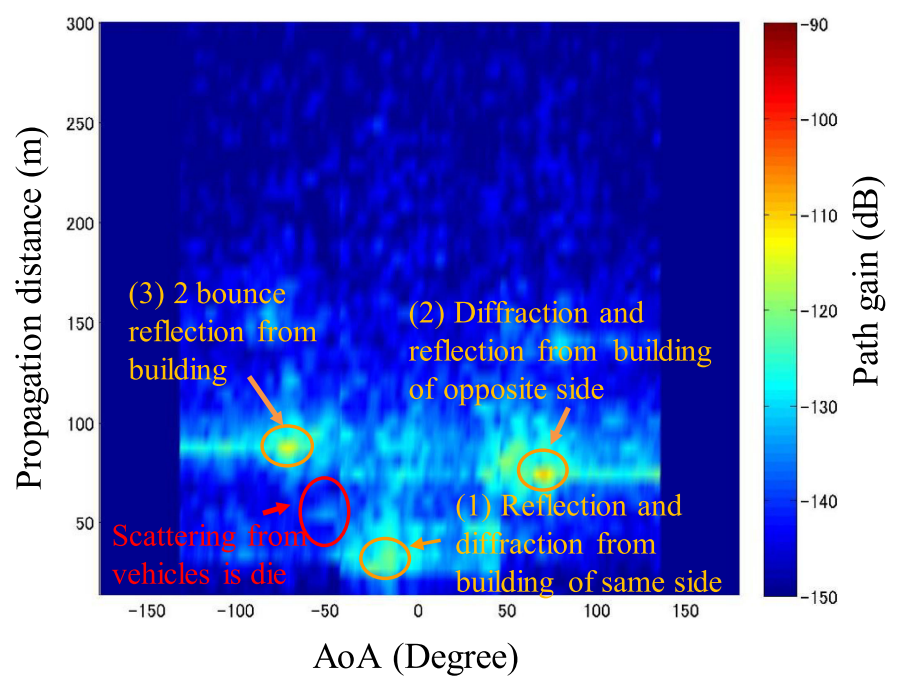

(c) Power angular delay profile at snapshot 3

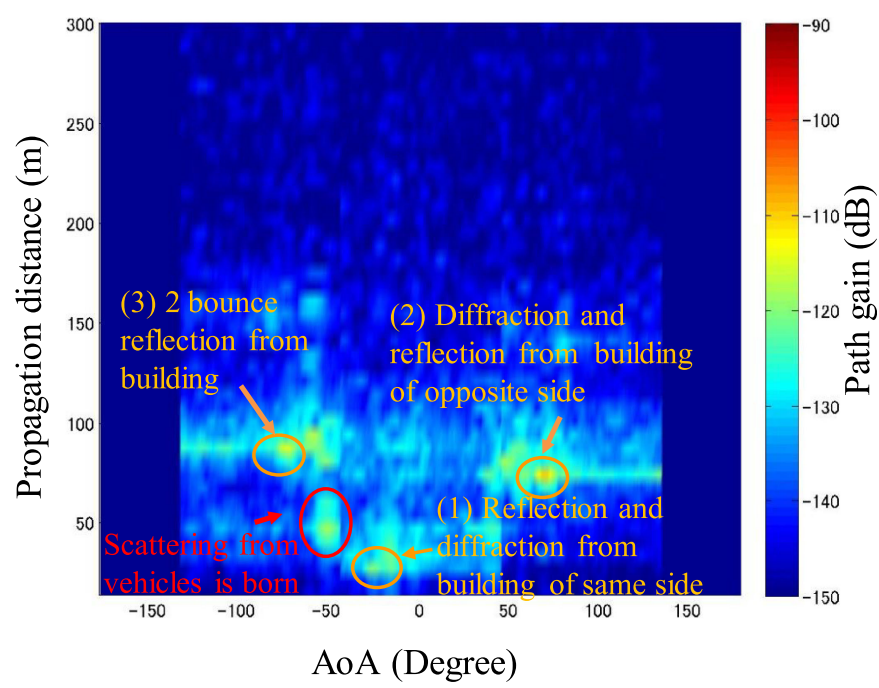

(d) Power angular delay profile at snapshot 4 


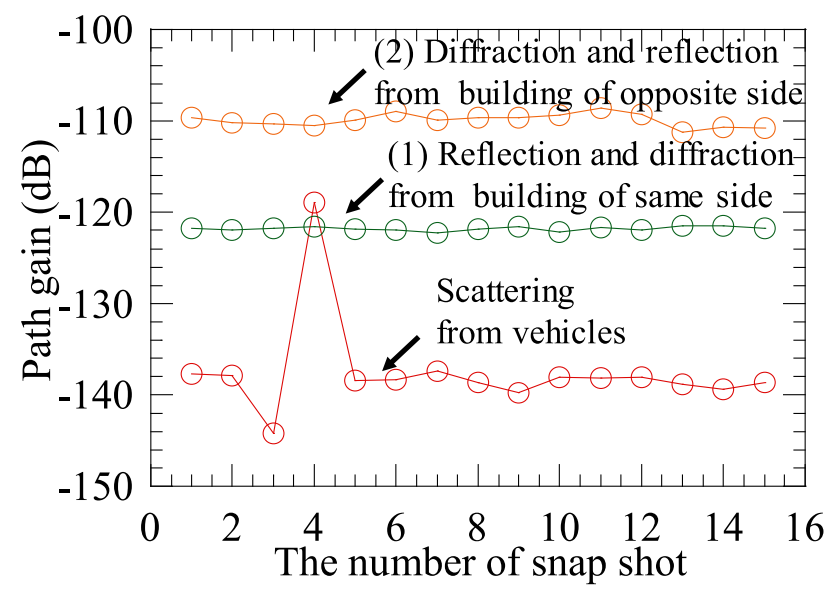

(e) Time fading characteristics of received power at propagation distance of 47 $m$ and arrival angle -50 degrees.

Fig. 2. Measurement results.

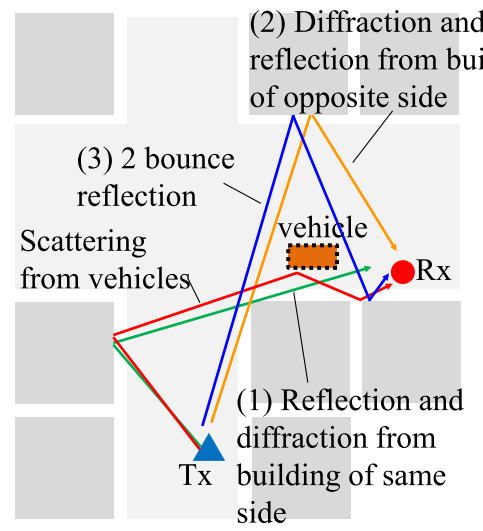

Fig. 3. Dominant paths in NLOS urban environment.

\section{Conclusions}

In this paper, we clarified the dynamic channel properties based on diffuse scattering from vehicles for high frequency bands in a NLOS urban environment. We investigated the dominant paths based on the measured power delay angular profile using a 20-GHz band channel sounder. We confirm based on these results that the arrival waves from the building on the same or opposite side of the Rx antenna and the scattering path from the vehicles on the road are dominant in a NLOS urban environment. We also found that the fading of the scattering paths is approximately $26 \mathrm{~dB}$ in those paths when they are born and die. In order to design a service area, constructing a prediction method considering scattering from vehicles on the road will be the subjects for our future work.

\section{Acknowledgments}

This paper includes a part of the results from "The research and development project for realization of the fifth-generation mobile communications system" commissioned by The Ministry of Internal Affairs and Communications, Japan. 